\title{
Joint Video Self-Modeling as a Conversational Intervention for an Individual with Traumatic Brain Injury and His Everyday Partner: A Pilot Investigation
}

\author{
Jerry K. Hoepner, Sarah E. Olson \\ Department of Communication Sciences and Disorders, University of Wisconsin, Eau Claire, Wisconsin, United States
}

\begin{abstract}
Purpose: This investigation examined the effects of joint video self-modeling (VSM) as an intervention for social interactions by individuals with traumatic brain injuries (TBI) and their close communication partners.
\end{abstract}

Methods: One person with TBI and his everyday communication partner attended 16 intervention sessions, jointly reviewing recordings of conversations from their home and community under the guidance of a coach. Self-reflection was prompted using a hierarchy of prompts ranging from open-ended to direct. Sessions were transcribed and coded based on the conversational purpose of each statement. A mixed methods design was used to collect quantitative and qualitative outcomes.

Results: Participants generated appropriate self-assessments of communication behaviors when viewing recorded interactions following prompts from a coach. Pre- and post- measures of social communication identified improvements in self-awareness and self-regulation. While partner' LaTrobe Communication Questionnaire remained stable, ratings increased for the participant with TBI, indicative of increased self-awareness. Measure of Participation in Conversation (MPC) scores for interaction and transaction both improved for the participant with TBI. Likewise, Measure of Skill in Supported Conversation (MSC) scores for acknowledging and revealing competence increased for the partner. Seven broad categories of discourse statements emerged.

Conclusions: While this investigation is preliminary, VSM appears to hold potential for increasing self-awareness and improving communication interactions for individuals with TBI and their partners. A reduction in less desirable behaviors by the individual with TBI suggests that prompting self-assessment may foster self-regulatory behaviors. The partner increased positive conversational supports and a reduced set-ups and antagonistic statements.

Keywords: Traumatic brain injury, Video self modeling, Communication partner training, Technology, Self-regulation, Self-awareness

\section{INTRODUCTION}

Impairments in social discourse and self-awareness often compromise exchanges between individuals with traumatic brain injuries (TBI) and their everyday partners [1,2]. Individuals with TBI present with poor presupposition, unclear referents, problems initiating and maintaining topics, topic repetitiveness, interruptions, tangential topic shifts, verbosity or terseness, vague or overly specific explanations, perseverations, and other inappropriate social behaviors [3-9]. Those pragmatic impairments break social

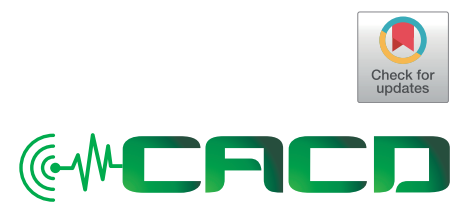

Received: February 1, 2018

Revision: April 17, 2018

Accepted: April 20, 2018

\section{Correspondence:}

Jerry K. Hoepner

University of Wisconsin, Eau Claire, 105 Garfield Avenue, Eau Claire, Wisconsin, United States Tel: +715-836-3980 Fax: +715-836-4185 E-mail: hoepnejk@uwec.edu
(C) 2018 The Korean Association of SpeechLanguage Pathologists

This is an Open Access article distributed under the terms of the Creative Commons Attribution NonCommercial License (http://creativecommons.org/ licenses/by-nc/4.0/) which permits unrestricted noncommercial use, distribution, and reproduction in any medium, provided the original work is properly cited. 
norms, placing a strain on their communication partners [1,2]. Typically, individuals with TBI are less aware of cognitive, emotional, and social impairments than of more concrete and observable physical impairments [10-13]. Such impairments contribute to less rewarding, less interesting, and more effortful interactions for everyday partners [14].

Angeleri and colleagues [15] argue for a broader description of pragmatic impairments that extends beyond linguistic elements to include extralinguistic, paralinguistic, context, and conversational elements. Paralinguistic impairments include difficulties comprehending vocal tone and prosodic elements. Extralinguistic elements such as comprehension and expression of facial expression are also impaired [16,17]. Difficulties with interpreting facial emotions can affect one's ability to interpret feelings and compromise social communication competence [18]. Further, such individuals struggle to recognize emotional expression in voice $[17,19]$. Impairments in their ability to interpret emotional intent through facial expression, along with tone and prosody of voice, may be at the heart of the social impairments [20].

A broader conceptualization is important since individuals with TBI are likely to have better self-awareness of more observable functions related to initiation and generation of language (e.g., word finding, voice amplitude) than less tangible social factors $[6,21,22]$. Grice [23] identified four maxims for typical discourse, which serve as a framework for considering social communication impairments in TBI. Typical discourse includes appropriate quantity of information, quality (accuracy and truthfulness), relation (adding information that is relevant to the topic), and manner (clarity and lack of ambiguity). Individuals with TBI frequently violate these maxims, which results in disruptions to conversational discourse [6]. Many believe that these conversational difficulties are the result of underlying cognitive impairments [24-26]. Disorders of executive functions, including limitations in working memory, are context dependent. As such, the level of environmental (both the physical and partner environment), task, and internal demands (e.g., pain, fatigue, hunger, anxiety) can have an impact on conversational performance. Therefore, adjustments to the environment, task demands, and internal thought processes have the potential to reduce or ameliorate some social communication difficulties.

While partners may intuitively make some adjustments that support an individual with TBI, not all necessary changes are implicit. Body and Parker [3] examined the interactions between an individual with TBI who displays topic repetitive- ness and everyday partners, identifying some partner behaviors that effectively redirect but also some behaviors that reinforce the repetitive topic. This indicates that behaviors such as topic repetitiveness may be jointly constructed, which suggests that partners need to adjust their interactions. This is a difficult context to navigate, as effective partners must be selective in their interaction approaches. Partners must use more prompting, topic maintaining, and redirecting, while experiencing less enjoyable, interesting, rewarding, and socially reinforcing interactions $[4,14,27-30]$. As such, individuals with TBI place an increased effort and burden upon their partners, especially in maintaining the flow of conversation $[4$, 14]. Further, the lack of social reinforcement of partners by individuals with TBI provides fewer opportunities to share personal interests, less time to make social connections, and places strains on their relationships $[1,2]$. If partners do not make adjustments, conversations are hard to follow, often disconcerting, and uncomfortable [14,29]. When partners do make adjustments, problematic behaviors are reduced, however; perceptions of burden among partners increase as well [29].

A challenge for interventions serving individuals with TBI and their everyday partners relates to inaccuracies in assessment of self and others. Individuals with TBI tend to underestimate their struggles and overestimate their performance [6, $31,32]$. Compared to their partners, individuals with TBI underreport emotional and non-verbal communication problems such as tone of voice, responsiveness to social cues, and their own facial expressions [33]. Conversely, partners may be overly critical of their loved ones with TBI, over-identifying problems and underestimating abilities. These judgments are further compromised when they take place outside of the context of an interaction. Retrospective judgments are subject to a negativity bias, where challenges are more likely to be identified than strengths [34]. Cumulative biases are also common in close partner relationships, leading to an over identification of challenging behaviors $[35,36]$. Reducing demands on retrospective recall likely increases accuracy of judgments by the individual with a TBI and their everyday partner. Hoepner and Turkstra [37] found that direct video review of self- and partner-assessments using the La Trobe Communication Questionnaire (LCQ) produced improved consensus on both effective and challenging interactional behaviors. Identifying consensus, in this manner, may provide an effective starting point for joint interventions addressing conversational interactions. Increasing the awareness of indi- 
viduals with TBI appears to be critical to their continued recovery. Those with more impaired metacognitive knowledge are less motivated [38]. Further, they are less effective in using compensatory strategies and may disregard treatment recommendations [39,40]. Finally, those who have better awareness have better employment outcomes [41].

Existing interventions for self-regulation provide a context for reviewing one's own performance in real life contexts. Metacognitive strategy instruction (MSI) [42-48] employs direct instruction to train individuals with TBI to regulate their own behavior by breaking complex tasks into steps and thinking strategically. Ownsworth and colleagues $[49,50]$ have used this approach within cooking tasks (among other functional contexts like volunteer and work tasks), to increase awareness of errors and foster self-regulation. Generally speaking, these approaches change awareness and regulation within tasks but do not generalize broadly to an individual's global self-awareness and regulation. Following an obstacle-goal-plan-do-review (OGPDR) [51] framework, an individual with TBI sets goals, predicts performance on real-world tasks, identifies best potential solutions drawing upon past performance, selfassesses during the activity, makes strategic adjustments, and evaluates performance after the activity. Similarly, time pressure management [52] practices real-life tasks under incrementally increasing attentional and time demands. Unfortunately, when not coupled with direct video support, self-assessments and reflections are subject to retrospection and memory failures. As a result, accuracy, specificity, and flexibility of judgments are likely to decline. A clinical outcome of such approaches is that many judgments and reflections are made by the clinician, rather than the clients.

Recognizing the challenges individuals with TBI and partners face when asked to make retrospective judgments, some interventions have employed direct video review. Ylvisaker [51] referred to these as self-coaching videos. Self-coaching videos allow individuals with TBI to see their successes and unsuccessful moments. Togher and colleagues [53] video recorded conversations between individuals with TBI and their communication partners. This allows for direct review of footage, which allows the individual with a TBI and his or her partner to see what they did right and wrong first hand [53]. Investigators found that joint intervention was superior to solo interventions, where addressing a jointly constructed conversation is less feasible. Youse and Coelho [54] used a similar video-review approach. In order to avoid a conversation that feels awkward or forced, interpersonal process recall
(IPR) [54] is used to facilitate the interaction within guided review of videos. IPR capitalizes on authentic interpersonal interactions and footage is reviewed immediately afterwards, fostering identification of insights related directly to the interaction dynamics. This technique improved situational anxiety, overall self-concept, interpersonal communication skills, and specific behavior associated with effective interpersonal communication [54]. Further, the authors noted that participants experienced improvements in day-to-day interactions supporting generalization of skills developed through IPR. Review of the video allows individuals with TBI and their partners to see what they could have done better, along with things that went well.

Video self-modeling (VSM) is another intervention that capitalizes on direct review of video footage to provide a tangible context for judgments about one's own performance. A substantial volume of empirical evidence exists regarding the use of VSM across various disciplines and clients [55-61]. The utility of VSM has been examined among English language learners, adolescents and adults who stutter, children with Asperger's syndrome, individuals with autism spectrum disorders, individuals with cognitive disabilities, individuals with reading disabilities, children in educational settings, and individuals with traumatic brain injury. It has been used to improve behavioral interactions among individuals with disabilities. Everyday partners have been examined in parent-child dyadic contexts [62,63]. For an exceptional review of past VSM research, see Buggey and Ogle [56]. Early conceptualizations of video self-modeling constrained direct video review to instances of positive behaviors [64]. The process began with video recording of an interaction, followed by examination of the interaction to identify segments of positive/successful behaviors, parse out those segments and eliminate any maladaptive instances, and then bring the footage back to the session for guided review. Constraints of editing and playback reduced accessibility and feasibility of this approach. The advent of smart technologies have made the process of recording, selecting, and reviewing more transparent and accessible. It also opens up the possibility of reviewing video without editing out challenges, a modification to standard VSM. Buggey and Ogle [56] would likely contend that video review approaches that capitalize on identifying challenges as well as successes are distinctly different than VSM. In principle, a modified approach need not be substantially different, as the coach has the ability to dictate the balance of challenges and successes prompted. Further, the eventual goal is to move to- 
wards more identification of successes. Limiting guided review to positive instances may constrain the potential value and feasibility of VSM. The primary impetus behind the principle of identifying positive instances is grounded in errorless learning and positive feedback. By facilitating identification of both successes and challenges, coaches can still foster positive feedback while increasing awareness and fostering selfregulatory behavior. If a challenge is identified, such as "I interrupted my wife," the clinician responds with positive feedback such as "You did interrupt your wife but it is good that you are starting to recognize that." Hoepner, Sell, and Kooiman [65] examined modified VSM and video other-modeling as a way to train an everyday partner. Importantly, they identified implicit changes to the partner's interactions, which included reduced demands and quizzing behavior, while increasing use of supported conversation behaviors. That modified approach prompted assessments for both interactional successes and challenges.

The modified VSM procedure capitalizes on reviewing footage to foster awareness of both interactional participants. While review of the client and partner interactions occur jointly, judgments about the successes and challenges for the client and the partner should occur separately. In other words, the client should make the judgments of his/her own interactions and the partner should make judgments of his/ her own interactions. Coaches should refrain from making judgments about a client's interaction (i.e., successes and challenges). However, the coach should scaffold and evoke a self-assessment. Once the person makes an assessment, the coach can reframe, reiterate, or restate each individual's assessment. This approach draws heavily on the principles of motivational interviewing [66]. A primary tenet of motivational interviewing is to resist the "righting reflex," a tendency to answer or provide a solution for another individual. Rather, clinicians use open-ended questions to elicit the client's own potential solutions, affirming appropriate suggestions, reflecting upon the process, and summarizing the individuals' statements of planning (OARS: open-ended questions, affirmation, reflection, and summarization). In this manner, judgments are self-identified, reducing tendency for defensive resistance and dismissal. Another key element of motivational interviewing is the concept of encouraging acceptance of incremental change. While early self-assessments may not parallel accuracy or specificity of the assessments made by partners or coaches, awareness is improving.

The present investigation sought to address the following questions, while examining feasibility of such an approach in everyday clinical contexts:

1) Is joint, guided reflection of interactional behaviors through video self-modeling effective in changing the behaviors (awareness) of the person with TBI and the close partner?

2) Does that change in behaviors/awareness translate into better interactions?

The authors anticipated a change in self-regulation of behaviors as awareness of behaviors improves, resulting in more successful interactions.

\section{METHODS}

A mixed qualitative and quantitative design was employed, in order to provide detailed descriptions of the intervention approach and participant outcomes. This enabled investigators to address overlapping elements of each phenomenon [67]. Because this was a qualitative-dominant mixed method, investigators used frequency and distribution to validate interpretations stemming from qualitative coding. The feasibilitydriven design used here aligns with principles identified by Sohlberg and colleagues [68] intended to foster adjustments to protocols given clinical decision making. Rather than holding rigidly to a protocol, clinical decision making guides inthe-moment adjustments to meet the needs of the participants. This study was approved by the Institutional Review Board for the Protection of Human Subjects at the University of Wisconsin - Eau Claire, protocol 19462014.

\section{Participants}

An individual with a TBI and his spouse were recruited for this 16-week intervention study. For the purpose of this paper, fictitious names have been applied to foster a more humanized description. Joe was a 53-year-old male who sustained a moderate to severe TBI 27 months prior to initiating this intervention. Joe currently delivers daily newspapers and sells scrap metal as a way to contribute to the fiscal needs of his family. Joe's baseline communication behaviors were characterized by angry emotional verbal outbursts, emotional rants, statements of self-loathing, blaming others for his challenges, disproportionate upsets, and speaking loudly regardless of emotion. Joe identified his goals through an informal interview, including a desire to address the following: raising his voice, arguing with or yelling at others, and memory problems. For summary of Joe's demographic information and 
baseline testing scores, see Table 1. Sharon was a 50-year-old female spouse who works at a local newspaper. Sharon's baseline communication behaviors were characterized by frequent demands for recall of events, contributions to perseverative topics, and traps/set-ups that tended to exacerbate Joe's maladaptive communication behaviors. Sharon identified the following goals through informal interview: being less reactive to Joe's upsets and comments, avoiding questions that are likely to cause an upset, and providing an alternate means of response/memory supports. She also recognized her tendency to set-up or sabotage Joe at times, which typically resulted in further blow-ups.

\section{Characterization of participant status}

Initial cognitive status was measured by the Repeatable Battery for Assessment of Neuropsychological Status (RBANS) [69] as outcomes correlate strongly with measures of intelligence and memory. The RBANS has strong internal reliability and concurrent validity for use with adults with moderate-severe TBI [70]. RBANS total scores, subscale scores, and percentile ranks for pre-intervention are shown in Table 2. Likewise, Table 3 identifies scores, subscale scores, and percentile ranks post-intervention. Table 4, 6-month follow-up scores, are included to demonstrate the lack of stability in scores. Fluctuations in attention and memory scores may be indicative of factors related to timing and internal demands, as Joe frequently engaged in maladaptive self-talk throughout the assessments.

\section{Materials and procedures}

Interactions were recorded using the PDS Intelligent Stream Recording system at the University of Wisconsin - Eau Claire Communication Sciences and Disorders Center for Communication Disorders (CCD). A Sony digital audio recorder (ICDSX733) was used to ensure adequate audio fidelity for transcription. An iPod touch device (16 GB Apple 6th generation iPod touch) was used for recording home conversations. Recorded videos were reviewed directly under the direction of coaches. The second author served as the primary coach, leading 14/16 sessions with the first author serving as coach for the remaining two sessions. All iPod videos were downloaded to a secure drive, accessible only to the researchers. The La Trobe Communication Questionnaire (LCQ) [71] was used to examine perceived frequency of troublesome social discourse behaviors, following Hoepner and Turkstra's [37] method of direct review (i.e., participants viewed a video re-

Table 1. Demographic characteristics of participant with TBI

\begin{tabular}{lcccccccc}
\hline $\begin{array}{l}\text { Participant } \\
\text { with TBI }\end{array}$ & Age & $\begin{array}{c}\text { Months } \\
\text { post-injury }\end{array}$ & Coma (day) & Etiology & $\begin{array}{c}\text { Length of IP } \\
\text { stay (day) }\end{array}$ & $\begin{array}{c}\text { RBANS total } \\
\text { scores* }\end{array}$ & $\begin{array}{c}\text { LCQ total } \\
\text { scores* }\end{array}$ & $\begin{array}{c}\text { Sex } \\
\text { Joe }\end{array}$ \\
\hline
\end{tabular}

MVC, motor vehicle crash; IP, inpatient.

*Note that RBANS refers to the Repeatable Battery for Assessment of Neuropsychological Status and LCQ refers to the LaTrobe Communication Questionnaire.

Table 2. RBANS subscale scores on version A at pre-intervention

\begin{tabular}{lcccccc}
\hline & Immediate memory & Visuospatial/Construction & Language & Attention & Delayed memory & Total scale \\
\hline Index score & 57 & 105 & 82 & 88 & 75 & 77 \\
Percentile & 0.2 & 63 & 12 & 21 & 5 & 6 \\
\hline
\end{tabular}

Table 3. RBANS subscale scores on version B at post-intervention

\begin{tabular}{lcccccc}
\hline & Immediate memory & Visuospatial/Construction & Language & Attention & Delayed memory & Total scale \\
\hline Index score & 73 & 96 & 87 & 94 & 71 & 80 \\
Percentile & 4 & 39 & 19 & 34 & 3 & 9 \\
\hline
\end{tabular}

Table 4. RBANS subscale scores on version $D$ at six-month follow-up

\begin{tabular}{lcccccc}
\hline & Immediate memory & Visuospatial/Construction & Language & Attention & Delayed memory & Total scale \\
\hline Index score & 81 & 78 & 79 & 88 & 64 & 72 \\
Percentile & 10 & 7 & 8 & 21 & 1 & 3 \\
\hline
\end{tabular}


corded conversation of themselves and made ratings based on that interaction). Participants rate frequency of common problematic communication behaviors. The Togher and colleagues [53] modified versions of the Kagan scales [72]: modified Measure of Supported Conversation (mMSC), and modified Measure of Participation in Conversation (mMPC) were used to rate the nature of the dyad's interactions. Scoring was completed by two blinded clinicians who review video recorded conversations, using the scale anchors to rate subscales of client and partner behaviors.

\section{Pre-intervention conversation}

The couple engaged in a 10-minute conversation at the CCD following an open-ended prompt. The conversation was recorded using an iPod so that dyads could directly review their conversations using the LCQ immediately after the conversation. LCQ ratings by the person with TBI and close partner serve as an indication of perceived conversational success. Dyad review of the initial conversation using LCQ is used to generate consensus on conversational/interactional goals. Initial ratings of self for Joe produced a total score of 84 , whereas close other rating by Sharon was 64 . Note that Joe rated himself as more impaired than his spouse, Sharon.

Baseline dyadic conversations were transcribed and rated, using the modified Measure of Participation in Conversation (mMPC) and Measure of Supported Conversation (mMSC) [53]. Raters had access to the transcript and the video-recorded conversation. The mMPC is composed of two components, Interaction and Transaction .

The mMPC provides several anchor elements to support inter-rater reliability of ratings. Subsequently, all ratings were within 0.5 points of each other. During the baseline conversation, clinicians rated Joe's participation in the conversation as a 2.5/4.0 for Interaction. Rater one and rater two concluded identical ratings for interaction. Interaction is a measure of how the person with TBI engages within the conversation. While he does several things well such as adding to and maintaining topics, asking follow-up questions and clarifications, actively listening, and choosing appropriate topics, he frequently interjects, has several verbal blow-ups where he raises his voice, and has poor eye contact and facial expression when thinking (i.e., looks strained or as if he's thinking rather than engaging). Several times, he expressed frustration over his struggle to express his thoughts clearly. Likewise, baseline ratings of Joe's Transaction score was 2.0/4.0. Transaction is a measure of conversational productivity or contributions.
While he did several things well, such as maintaining an exchange of information, he was inconsistent in asking for clarification and utilizing supports. Organization of his thoughts was poor and at times he appeared to get lost in the conversation. The MSC is also composed of two parts, Acknowledging Competence and Revealing Competence. Clinicians rated Sharon's Acknowledging Competence as a strength, 3.0/4.0. Rater one and two provided identical ratings. Acknowledging Competence is a measure of a partner's regard for the competence of their loved one with TBI. In other words, the extent to which their words and actions show that they believe their loved one is competent and worth interacting with. This was positive given her soft spoken, methodical interaction, limited reciprocal response to Joe's upsets, and steady reassurance/ enthusiasm. Most of her talk was collaborative, rather than directive. At times, she was a bit dismissive of his upsets, not just redirecting and moving forward, but discounting. Revealing Competence was rated a 2.17/4.0, indicating some challenges with this construct. Revealing Competence includes techniques used to ensure a means of response and effective participation. While she gives cues in a conversational manner, introduces topics of interests, and allows Joe to take conversational turns, she frequently jumps back and forth between conversational topics in a manner the person with TBI cannot follow. When Joe encountered upset because he did not know how to respond to a question, she sometimes barked back with "I just asked!" Further, she did little to verify information through an alternative modality when Joe was clearly struggling to comprehend.

\section{Intervention}

The couple attended joint intervention sessions once a week for 16 weeks. During each 50-minute session, they reviewed conversations generated in their home and community using a guided, video self-modeling approach. Each week they were instructed to record 3 conversations that were at least $5 \mathrm{~min}$ utes in length (see Figure 1 for the joint video review protocol). Conversations were recorded within the couple's home and/or community, capitalizing on authentic interpersonal interactions [54] and the influence of authentic environmental contexts and interactional routines where conversations typically occur [51]. Participants were allowed to self-select recording contexts. Recorded interactions included making dinner together, waiting in the car for appointments, meal times, and spontaneous conversations. It is worth noting that participants identified these homework video contexts as 

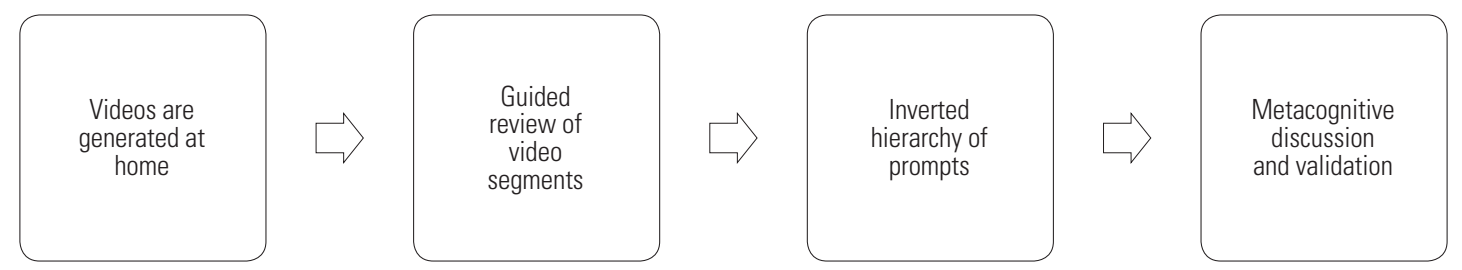

Figure 1. Joint video review protocol.

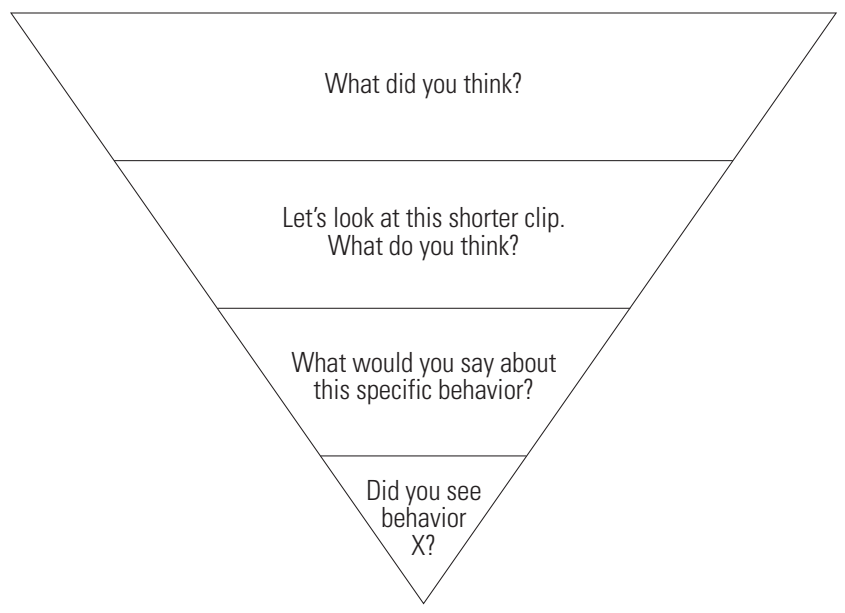

Figure 2. Inverted hierarchy video self-modeling protocol.

more authentic and natural than the planned conversations used for pre- and post-intervention videos. In the session, dyads jointly viewed those previously recorded conversations, guided by their previously identified goals. The coach initiated playback of a segment of the video, typically pausing the video immediately after an instance of interactional success or challenge. Eventually, the routine of pausing the video became a cue for the interactional behavior to be identified. Prompts followed an inverted hierarchy, beginning with a non-specific, open-ended prompt (see Figure 2 for the inverted hierarchy framework). If the participants are not able to identify the targeted interactional behavior given the video sample, the coach could replay the segment and/or shorten the video clip as a means of drawing attention to the success or challenge. The video review was intended to errorlessly prompt an assessment [51]. Therefore, if a self-assessment was not garnered after review of a shortened segment, the coach could provide a target-constrained prompt (e.g., "We have been talking about moments when you raise your voice. Let's view that segment again."). Finally, if the target-constrained prompt did not elicit a self-assessment, a direct target prompt could be used (e.g., "Did you raise your voice?"). Note that both the individual with a TBI and the partner were prompted to make

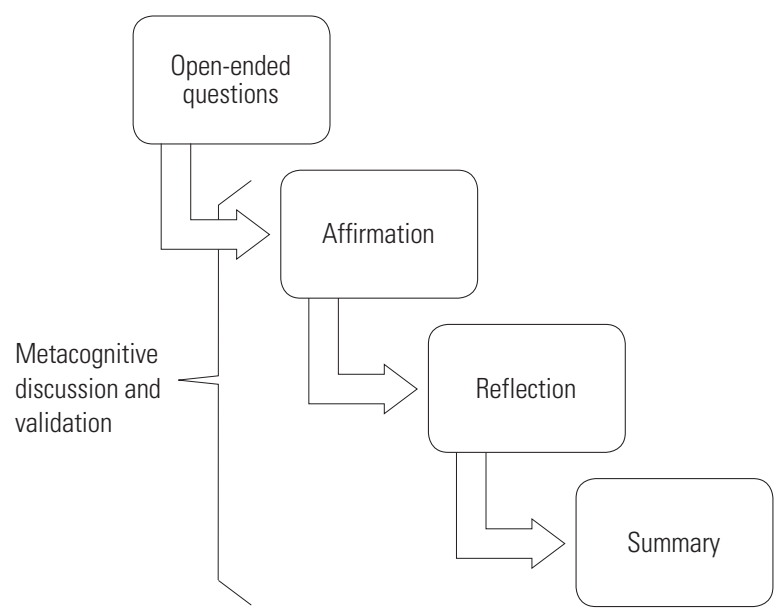

Figure 3. Reflection and motivational interviewing process.

judgments. Those judgments were only of themselves, not the other person. Note that judgments were all verbalized. After identifying successes and challenges from the video review, a coach facilitated discussion of their perceptions, highlighting areas of consensus and encouraging conversations about disagreements. This stage of the process drew heavily upon the principles of motivational interviewing [66] to foster self-identification of performance (see to Figure 3 for the process). At the discretion of each coach, dyads were directed to review videos generated in previous sessions, as a way to foster metaawareness of progress. While the second author was the primary coach (coach 1), the first author and primary investigator, who trained the second author and coach, served as coach for two sessions (coach 2).

\section{Post-intervention conversation}

Ten-minute dyadic conversations were elicited for post-testing measures, following the protocol previously described. Again, dyads evaluated perceived conversational success using the LCQ under direct review of the conversation. Transcriptions of the conversation, as well as video recordings, were rated by two blinded clinicians, using the mMSC, and mMPC. 


\section{Analyses}

Quantitative analyses included frequency counts and descriptive statistics. Frequency counts were tabulated for coach prompts, using the following levels based on the inverted hierarchy: L1 (i.e., open-ended prompt), L2 (i.e., time-constrained prompt), L3 (i.e., time and target-constrained prompt), and L4 (i.e., direct target prompt). Responses to prompts were also tabulated, allowing for generation of ratios for specific responses to prompts and prompt-levels. On target judgments were compiled for each individual (i.e., Joe, Sharon, coach 1, coach 2) within and across sessions. Frequency counts were also compiled to track the nature of on target judgments over time, as a way to examine identification of challenges and successes. Treatment fidelity was calculated based on the inverted hierarchy, assuming progression from least to most constrained prompting. This was jointly conducted by the first and second authors for each recorded session.

Qualitative coding was conducted for each of the 16 intervention sessions. A research assistant manually transcribed each of the sessions verbatim, identifying the speaker and including parenthetical notations for extralinguistic communication. Note that two of the coding categories were established a priori, prompts and judgments. The investigators made the decision to include them in qualitative coding as they were an inherent, contextual component of the interactional transcripts and required analysis for type (i.e., level of prompting specificity and nature of judgment). Manual, open and axial coding methods [73] were used to characterize the nature of each codable statement. The second author identified an initial code for each of the statements. Operational definitions of each code were established to ensure coding consistency. Next, the first and second authors reviewed each of the coded statements, negotiating best fit and merging similar codes through modifications of operational definitions when pertinent or validating existing codes given coder agreement. After consensus coding was completed, a third round of coding was completed to ensure complete consensus on each of the coded statements. As the process of consensus coding suggests, agreement was negotiated on all statements. Common discourse terms and conventions were used to categorize types of discourse within the transcripts. While they were not determined $a$ priori, the investigators used commonly used terms when possible. Many interactional elements were somewhat unique to the context of this intervention and were arrived at through a rigorous qualitative coding process.

\section{RESULTS}

\section{Cognitive and communication status measures}

Joe's performance on the baseline and follow-up RBANS assessments revealed inconsistencies across domains of cognition across the three intervals of assessment: pre-, post-, and follow-up (see Tables 2, 3, and 4). Total scores remained stable: baseline was 77 (6th percentile), post-intervention was 80 (9th percentile), and six-month follow-up of 72 (3rd percentile). That pattern of performance is consistent with attention impairments. No clear evidence of improvement or declines could be noted. Performance in all domains of cognition was severely impaired.

\section{LCQ}

Post-intervention ratings of the LCQ were completed based upon direct review of a video recorded conversation between the dyad. Sharon completed the Close Other rating form, identifying a total score of 63 (baseline score was 64). Joe completed the Self-rating form, identifying a total score of 101 (baseline score was 84). Interestingly, Joe rated himself harshly on items that fall within the Disinhibition/Impulsivity and Conversational Effectiveness factors identified by Struchen and colleagues [22]. Typically, individuals with TBI are more likely to identify deficits in Initiation/Conversational Flow that are more tangible and observable [22].

\section{MMPC and MMSC}

Post-intervention ratings of the MMPC and mMSC were completed by raters, given written transcripts of the interactions and video recorded segments to support their interpretations. Post-intervention conversation resulted in a 3.0/4.0 Interaction rating compared to a baseline of 2.5/4.0. It is worth noting that the conversation began with a statement by Joe revealing upset at the outset "I'm already on the verge. ...She's talkin' stupid." However, after that disclosure, Joe maintained effective composure and was pragmatically appropriate. He frequently added information to the topic, used active listening by acknowledging Sharon's comments, and leaned forward, looking towards Sharon. He did tend to dominate the conversation but that may be a reflection on his partner's yielding interaction style, generally fostering his contributions over making her own. Transaction was rated as a 2.75/4.0, improved from 2.0 in the baseline conversation. Joe initiated stories about frustrating situations and sometimes raised his voice briefly but ultimately maintained composure despite 
clear frustration. Because he held the floor throughout much of the conversation, limited cohesion, disorganized thought processes, and poor referents to people within his stories was evident. MSC partner ratings for Acknowledging Competence were 3.125/4.0, improving slightly from 3.0 in the baseline conversation. Generally speaking, she was calm and soft spoken, often deferring to Joe to take the lead. However, she seemed somewhat resigned to struggle through the conversation and had low energy. Revealing Competence ratings were 2.88/4.0, improved from 2.17 at baseline. Strengths included use of redundancy and summary to support comprehension, making connections between topics of conversation and supporting topic transitions, and adding pertinent information to topics. Further, she framed verifications in question format to assure she understood his perspective. Challenges included balancing attempts to foster his contributions while maintaining her own to create a balanced interaction. Typically, she deferred to Joe and simply follows his lead. At times, she appeared disengaged or going through the motions.

Frequency counts were compiled across 10/16, 50-minute sessions resulting in over 4,783 coded statements. The first two sessions included only baseline measures, as did the final session. The eighth session was used solely to counsel Joe, as he reported a substantial change in his awareness. Upon prompting judgments regarding the first segment of video reviewed, he expressed that he was "a burden to his wife and family... it would have been better if he had not survived." He also stated "it was better before I knew how bad I was." No further attempt to review videos was made during that session. Part of the session was used to discuss meeting with his coun-

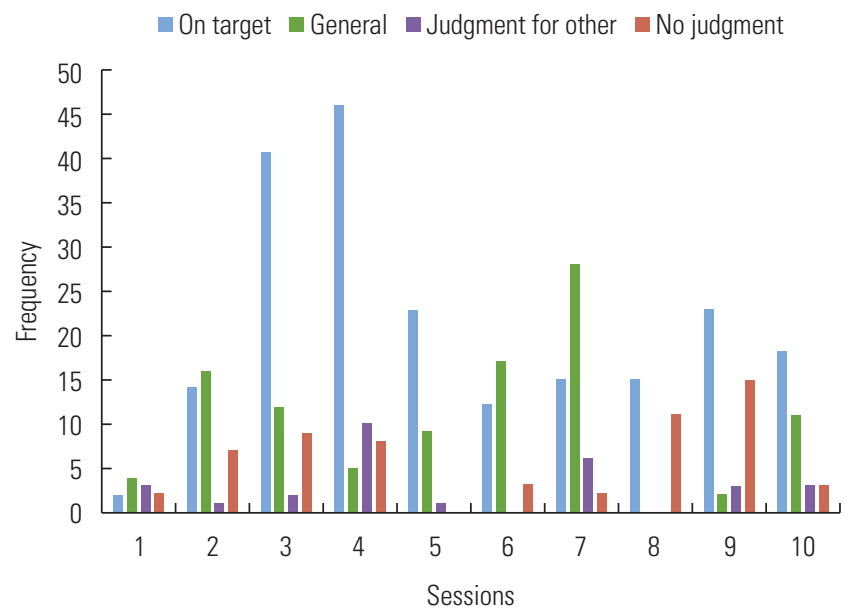

Figure 4. Distribution of number and type of judgments across sessions for Joe. selor. Recordings from two sessions were lost due to a technical problem with the video server.

The average of on-task time versus perseverations ratio was 9:1, based upon minutes on-task versus perseverations. The couple brought videos generated at home for $9 / 10$ sessions examined here. Joe made over 325 judgments total (out of 385 opportunities, 84\%), whereas Sharon made over 120 judgments total (out of 139 opportunities, $86 \%$ ). Note that making a judgment is different than making a judgment accurately, which will be discussed further. Only 96 judgments were made by the coaches, collectively. Figure 4 depicts the number and type of judgments per session for Joe, while Figure 5 displays number and type for Sharon.

Overall accuracy for on-target responses across 10 recorded sessions can be seen in Figure 6. Note that Joe reached an ac-

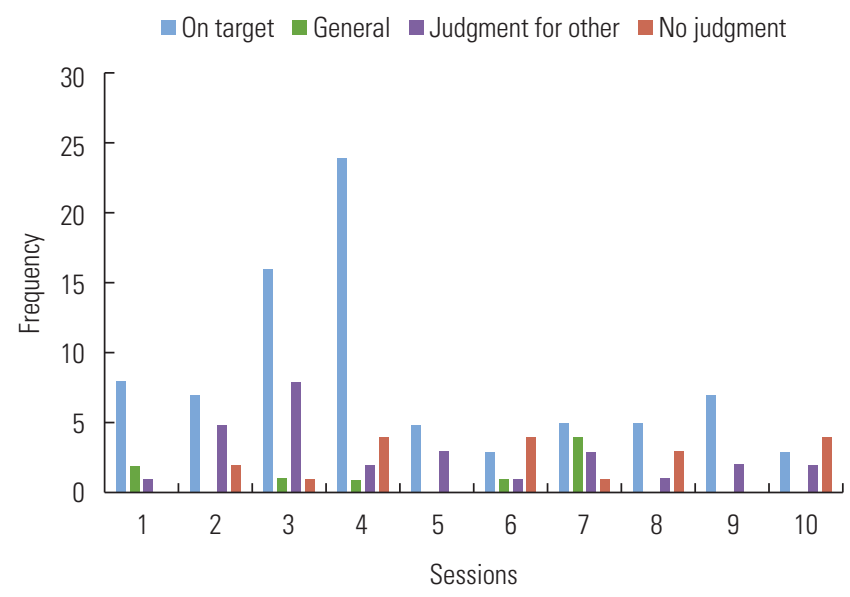

Figure 5. Distribution of number and type of judgments across sessions for Sharon.

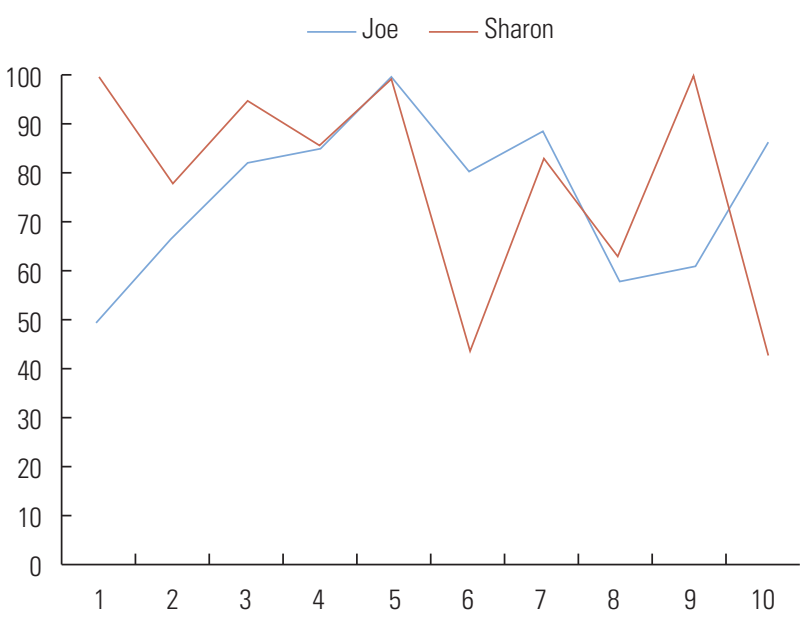

Figure 6. On-target judgments per recorded sessions. 
curate judgment for $75 \%$ of opportunities across all 10 recorded sessions and Sharon reached an accurate judgment in $82 \%$ of opportunities. While a relatively high level of accuracy was achieved, the number of prompts needed to reach an accurate response varied. Prompting sequences that ended in no judgment were considered inaccurate. Likewise, prompting sequences that ended in a judgment for other, with no on target judgment for self, were also considered inaccurate. Responses to L1 prompts (i.e., open-ended) were accurate $61 \%$ of the time (46/75). L2 prompted (i.e., time-constrained) responses were accurate only $29 \%$ of the time (4/18). L3 prompted (i.e., time and target-constrained) responses were $52 \%$ accurate (48/92). Responses to L4 prompts (i.e., direct target) were $59 \%$ accurate (52/88). Accuracy across prompt types was $55 \%$, meaning that multiple prompts were sometimes necessary to elicit an accurate self-assessment. Treatment fidelity for following incremental sequences of prompts that go from broad to more direct (e.g., L1-L2-L3-L4-judgment or L1-L2-judgment) was $29.27 \%$. Reasons for moving away from the rigid protocol will be addressed in the discussion section.

\section{Qualitative analysis}

Open and axial qualitative coding resulted in the development of seven categories and 21 subcategories. These categories were used to characterize the utterances of all participants (i.e., person with TBI, partner, and coaches that occurred during each session). Note that while interactions recorded at home or in the community were the basis for VSM review, the discourse within those conversations was not analyzed. Rather, the exchanges surrounding review of those videos was analyzed here. The seven categories identified included: a) prompting, b) judgments, c) maladaptive discourse behaviors, d) adaptive discourse behaviors, e) metacognition/metalinguistic, f) logistics, and g) uncodable utterances. Four levels of prompts were assigned $a$ priori (L1, L2, L3, and L4 described in detail in following sections). Judgments were further divided into subcategories of on target judgments, judgment for other, broad/general judgment, or no judgment. Maladaptive discourse behaviors were divided into the subcategories: perseveration on interaction, interruption, and off topic. Adaptive discourse behaviors included the following subcategories: validation, summary, clarification, response to clarification, elaboration, acknowledgement, redirection, attempt to close topic, and small talk. Finally, Metacognitive and Metalinguistic discussions included subcategories of memory/orientation to video, understanding and managing
TBI, explanation, and discussion of VSM approach. Note that some utterances were identified as logistics and the remaining statements were uncodable. See Table 5 for summary of coded elements for each participant and coach.

\section{Prompting}

Codes that fit within the category of prompting included all utterances that had the intention of prompting a judgment about one's own actions within an interaction. Joint review of video recorded interactions collected at home took place within each session. Prompts occurred upon pausing segments of video in order to elicit a judgment about the interaction. As such, all prompts were initiated by the coach. In total, 273 out of $4,783(6 \%)$ codable statements were coded as prompts, accounting for $14 \%$ of the coaches' codable statements. Four subcategories of prompts were observed: a) $L 1$, b) $L 2$, c) $L 3$, and d) $L 4$ (i.e., broadest to most direct within inverted hierarchy). Expanded descriptions of these subcategories are stated below.

a) L1. Out of the 273 prompting statements, 75 of those utterances fall within this subcategory (27\%). L1 prompts are defined as being broad, non-directional cues regarding previously watched video segment. An example of an L1 prompt is, "What did you think of that conversation?" Response accuracy was $61 \%$.

b) L2. This subcategory included 18 of the 273 (7\%) prompting category. An L2 prompt leads to further analysis of the video clip by replaying either the full clip itself or a segment of it, thus reducing the length of the clip. A commonly used L2 prompt was, "Well, let's listen to that again." Response accuracy was $29 \%$.

c) L3. L3 prompts made up 92 of the 273 (34\%) this category. These prompts asked about something specific about a targeted behavior within the interaction, but are not yes/no questions. An example is, "How do you think your tone of voice was in this clip?" Response accuracy was $52 \%$.

d) L4. This subcategory included 88 of the 273 (32\%) utterances that fall under the prompting category. An L4 prompt is the most specific prompt. It asks a yes or no question in regards to a specific action that occurred in the recorded video. "Did you raise your voice?" is an example of a basic L4 prompt. Response accuracy was $59 \%$.

\section{Judgments}

The judgments category contains all utterances that serve the 
Table 5. Frequency counts for coded statements per interactant.

\begin{tabular}{|c|c|c|c|c|}
\hline Cat. & Code & John & Sue & Coach \\
\hline \multirow[t]{5}{*}{ Prompts } & Total prompts & $0(0 \%)$ & $0(0 \%)$ & $273(14 \%)$ \\
\hline & L1 & NA & NA & $75(27 \%)^{*}$ \\
\hline & L2 & NA & NA & $18(7 \%)^{*}$ \\
\hline & L3 & NA & NA & $92(34 \%)^{*}$ \\
\hline & L4 & NA & NA & $88(32 \%)^{*}$ \\
\hline \multicolumn{5}{|l|}{ Judgments } \\
\hline & On target judgment & $209(11 \% / 54 \%)$ & $86(10 \% / 62 \%)$ & $0(0 \% / 0 \%)$ \\
\hline & Judgment for other & $29(1 \% / 8 \%)$ & $25(3 \% / 18 \%)$ & $92(5 \% / 96 \%)$ \\
\hline & General judgment & $87(4 \% / 23 \%)$ & $9(1 \% / 6 \%)$ & $4(<1 \% / 4 \%)$ \\
\hline & No judgment & $60(3 \% / 16 \%)$ & $19(2 \% / 14 \%)$ & NA \\
\hline & Percent of totals & $385(19 \%)$ & $139(17 \%)$ & $96(5 \%)$ \\
\hline \multirow[t]{4}{*}{ Maladaptive discourse behaviors } & Perseveration on interaction & $617(31 \% / 70 \%)$ & $300(36 \% / 88 \%)$ & $5(<1 \% / 12 \%)$ \\
\hline & Interruption & $199(10 \% / 23 \%)$ & $26(3 \% / 8 \%)$ & $3(<1 \% / 7 \%)$ \\
\hline & Off topic & $61(3 \% / 7 \%)$ & $13(2 \% / 4 \%)$ & $35(2 \% / 81 \%)$ \\
\hline & Percent of totals & $877(44 \%)$ & $339(41 \%)$ & $43(2 \%)$ \\
\hline \multirow[t]{10}{*}{ Adaptive discourse behaviors } & Validation & $37(2 \% / 8 \%)$ & $25(3 \% / 10 \%)$ & $185(9 \% / 14 \%)$ \\
\hline & Summary & $1(<1 \% /<1 \%)$ & $3(<1 \% / 1 \%)$ & $62(3 \% / 5 \%)$ \\
\hline & Clarification & $105(5 \% / 23 \%)$ & $14(2 \% / 6 \%)$ & $13(<1 \% / 1 \%)$ \\
\hline & Response to clarification & $8(<1 \%,<1 \%)$ & $58(7 \% / 23 \%)$ & $48(2 \% / 4 \%)$ \\
\hline & Elaboration & $191(10 \% / 42 \%)$ & $69(8 \% / 27 \%)$ & $11(<1 \% /<1 \%)$ \\
\hline & Acknowledgment & $40(2 \% / 9 \%)$ & 44 (5\%/17\%) & 738 (37\%/54\%) \\
\hline & Redirection & $3(<1 \% /<1 \%)$ & $3(<1 \% / 1 \%)$ & $155(8 \% / 11 \%)$ \\
\hline & Attempt to close topic & $0(0 \% / 0 \%)$ & $1(<1 \% /<1 \%)$ & $89(5 \% / 7 \%)$ \\
\hline & Small talk & $64(3 \% / 15 \%)$ & $36(4 \% / 14 \%)$ & $61(3 \% / 4 \%)$ \\
\hline & Percent of totals & $450(23 \%)$ & $253(31 \%)$ & $1,362(69 \%)$ \\
\hline \multirow[t]{5}{*}{ Metacognition \& Metalinguistic } & Memory/orientation to video & $173(9 \% / 64 \%)$ & 47 (6\%/59\%) & $5(<1 \% / 3 \%)$ \\
\hline & Understanding/managing TBI & $55(3 \% / 20 \%)$ & $12(1 \% / 15 \%)$ & 172 (9\%/91\%) \\
\hline & Self-explanation & $32(2 \% / 20 \%)$ & $16(2 \% / 20 \%)$ & $1(<1 \% /<1 \%)$ \\
\hline & Discussion of VSM approach & $10(<1 \% / 4 \%)$ & $5(<1 \% / 6 \%)$ & $11(<1 \% / 6 \%)$ \\
\hline & Percent of totals & $270(14 \%)$ & $80(10 \%)$ & $189(10 \%)$ \\
\hline Logistics & Logistics \& troubleshooting & $3(<1 \%)$ & $12(1 \%)$ & $12(<1 \%)$ \\
\hline 4,783 & & 1,985 & 823 & 1,975 \\
\hline
\end{tabular}

Operational definitions for each of the codes above are in Appendix A. Percentage of distribution per each code per individual is listed in parentheses followed by percentage of type per code. *Percentages identified are percent of total prompts.

purpose of describing the nature of one's behavior and make up 620 of the 4,783 (13\%) of codable statements. The four subcategories of judgments include: a) on target judgments, b) judgments for other, c) general judgments, and d) no judgment and are described below. A total of 385 (19\%) of Joe's codable statements were judgments, 139 (17\%) of Sharon's statements, and 96 (5\%) of the coaches' statements.

\section{a) On target judgments}

On target judgments are utterances that state observations about the speaker's actions in the video. These follow a prompt from the coach with the exception of instances where no judgment is made. On target judgments accounted for $54 \%$ $(n=209)$ of Joe's judgments, 62\% $(n=86)$ of Sharon's, and none were made by coaches (as judgments made for others 
were coded as Judgments for Others, regardless of accuracy).

\section{b) Judgments for other}

A judgment for other is a statement that describes the nature of another person's behavior or general events about someone other than the speaker. These made up 146 of the 620 (24\%) total judgments. Coaches made $63 \%$ of those judgments, which accounted for $30 \%$ of total judgments. Many of these occurred after prolonged prompting sequences without eliciting an on target judgment. Eight percent $(n=29)$ of Joe's judgments were made about his spouse, whereas Sharon made $18 \%(n=25)$ of her judgments about Joe. Note that $96 \%$ $(n=92)$ of coaches' judgments were made for either Joe or Sharon.

\section{c) General judgments}

General judgments composed 100 of the 620 (16\%). These judgments are about big-picture issues and are not prompted by the coach. They do not reflect directly on a video or interaction that occurred within the session, but on application of communication behaviors to everyday life or emotions in general. Twenty-three percent ( $\mathrm{n}=87$ ) of Joe's judgments, $6 \%$ $(n=9)$ of Sharon's, and $4 \%(n=4)$ of coaches' judgments were general judgments. It is noteworthy that Joe accounted for $87 \%$ of these statements.

\section{d) No judgment}

The no judgment subcategory includes 79 of the 620 (13\%) utterances within the judgments category. This subcategory contains statements or moments following a prompt where no interpretation of behaviors is made. Further, it may follow perseverations or off-topic discourse, where an opportunity to make a judgment remains and sometimes occurs. Examples include moments of silence following a prompt or statements such as "I don't know." Joe made no judgment in only $16 \%$ $(\mathrm{n}=60)$ of prompted opportunities, meaning his ability to make a judgment was $84 \%$ errorless. Similarly, Sharon failed to make a judgment in only $14 \%(n=19)$ of opportunities.

\section{Maladaptive discourse behaviors}

Utterances included within the maladaptive behaviors category are statements that do not relate to the topic of conversation and/or were inappropriate conversation behaviors. Maladaptive behaviors account for 1,031 of the 4,783 (22\%) codable statements. Seventy percent of those instances were made by Joe, while $27 \%$ were made by Sharon. Only $3 \%$ were made by coaches. There were three subcategories of maladaptive behaviors: a) perseveration on interaction, b) interruption, and c) off-topic. A perseveration on interaction is a statement that created a change of topic inappropriately or is a statement that elaborates on an irrelevant detail. This includes telling lengthy stories related or unrelated to the recorded interaction that do not enhance the discussion about behaviors seen within the recorded interaction, statements that describe a non-relevant topic, or statements that inappropriately return to a topic that was previously discussed. None of these statements include any form of judgment. Interruptions are present when a speaker interjects or overlaps with a partner's speaking turn. An off-topic statement is defined as a statement that does not relate to the subject of the conversation or the session in general. Maladaptive discourse accounted for $44 \%$ of Joe's total codable statements $(n=877)$. Similarly, maladaptive discourse accounted for $41 \%(n=339)$ of Sharon's codable statements. Note that coaches used $<2 \%$ maladaptive behaviors throughout codable statements. Seventy percent $(\mathrm{n}=617)$ of Joe's maladaptive discourse behaviors were perseverations on the interaction, while $23 \%(\mathrm{n}=$ 199) were interruptions. Eighty-eight percent of Sharon's maladaptive discourse behaviors were perseverations on the interaction $(\mathrm{n}=300)$, while $8 \%(\mathrm{n}=26)$ were interruptions. Off-topic behaviors accounted for $7 \%$ of Joe's maladaptive discourse, $4 \%$ of Sharon's, and $81 \%$ of coaches' maladaptive discourse ( $\mathrm{n}=35$ incidents). Note that those 35 incidents among coaches accounted for only $2 \%$ of their codable behaviors overall.

\section{Adaptive discourse behaviors}

Adaptive discourse behaviors are productive to the interaction and accounted for 2,065 of 4,783 (43\%) of total statements. See Table 5 for all values. Such behaviors accounted for $23 \%$ of Joe's codable interactions $(\mathrm{n}=450)$. Similarly, $31 \%$ $(n=253)$ of Sharon's codable statements were adaptive. Sixtynine percent $(n=1,362)$ of coaches' codable statements were adaptive discourse behaviors. Detailed below are operational definitions and examples for the nine subcategories of discourse behaviors:

\section{a) Validation}

Statements coded as a validation include 233 of the 2,008 (12\%) adaptive discourse behaviors utterances. Validations are statements that show agreement with something that another person said or simply serve as affirmation. Validation 
statements that typically occur are, "Right," "I agree," or "um hmm." These statements accounted for $8 \%$ of Joe's statements, $10 \%$ for Sharon, and $14 \%$ of coaches' adaptive discourse behaviors.

\section{b) Summary}

Summary statements include 66 of the 2,008 (12\%) total adaptive discourse behaviors. These statements give a brief outline of something that was recently discussed. Summary statements include summarizing what happened what was discussed that day at the end of each session as well as summarizing what was said in order to confirm understanding with all participants. Only a small portion of adaptive discourse behaviors included summary (Joe $\leq 1 \%$, Sharon $=1 \%$, coaches $=5 \%$ ).

\section{c) Clarification}

124 of the 2,008 (6\%) utterances within the adaptive discourse behaviors category fall under the subcategory clarification. These are statements or questions that ask about what was just said in order to remember or completely understand the topic. Examples of clarification include, "What did I say?" or "What was that again?" Such statements account for $23 \%$ of Joe's adaptive discourse, $6 \%$ for Sharon, and $1 \%$ for coaches.

\section{d) Response to clarification}

This subcategory includes 99 of the 2,008 (5\%) utterances within the discourse behaviors category. A response to clarification is defined as a statement that answers clarification questions or statements. While these were less than one percent of Joe's adaptive discourse behaviors and $4 \%$ for coaches, they account for $23 \%$ of Sharon's adaptive discourse.

\section{e) Elaboration}

Elaboration statements account for 271 of the 2,008 (13\%) discourse behaviors utterances. These statements add details to something that was previously stated. Such statements often follow judgments, when the individual making the judgment further explains that judgment. Nearly half (42\%) of Joe's adaptive discourse and $27 \%$ of Sharon's were elaboration. Less than one percent of coaches' adaptive discourse were elaborations.

\section{f) Acknowledgments}

This subcategory accounts for 820 of the 2,008 (41\%) discourse behaviors. Statements within this subcategory acknowledge another person's statement. Common examples include, "Okay," or "Mhm." Such statements account for $54 \%$ of coaches' adaptive discourse, along with $17 \%$ for Sharon, and $9 \%$ for Joe.

\section{g) Redirection}

The redirection subcategory contains 161 of the 2,008 (8\%) discourse behaviors utterances. These statements attempt to direct the conversation to the original topic. They typically follow a perseveration on interaction or off topic statement. Redirection statements directly leads back to playing a video or a prompt, whereas an attempt to close is not as direct. An example of redirecting is, "Mhm. Yeah. Should we take a look at the last video?" While only accounting for $1 \%$ of Sharon's adaptive discourse and $<1 \%$ for Joe, such behaviors make up $11 \%$ of coaches' adaptive discourse.

\section{h) Attempt to close}

These statements include 90 of the 2,008 utterances within the adaptive discourse behaviors category. An attempt to close is when one of the coaches or participants attempts to move on to a different topic and/or end the current discussion. Examples of these statements are "Well, that was a really tricky situation. Glad that everything turned out okay." These statements imply that this conversation should be over. They are primarily used by coaches (7\%), rarely by Sharon (1 instance), and were not used by Joe.

\section{i) Small talk}

Small talk includes 161 of the 2,008 (8\%) discourse statements. This subcategory is made up of statements that form natural chit-chat (e.g., the weather, recent events, scheduling, etc.). These statements occur at the beginning and end of each session before the intervention has officially started or after a motion to end the session has been made. This accounted for $15 \%$ of Joe's adaptive discourse, $14 \%$ of Sharon's, and $4 \%$ for coaches.

\section{Metacognition/metalinguistic}

The codes that fall within the metacognition/metalinguistic category relate to comprehension and reflection on the interaction. These statements can demonstrate increased awareness and understanding for the speaker and/or the listeners. The metacognition/metalinguistic category includes 539 of 4,783 (11\%) codable statements. Such statements accounted for $14 \%$ of Joe's codable statements and $10 \%$ for Sharon and coaches. This category is divided into 4 subcategories that are 
described below: a) memory \& orientation statements, b) understanding and managing TBI behaviors, c) self-explanation, and d) discussion of idea behind VSM.

\section{a) Memory and orientation statements}

These statements include 225 of 539 (42\%) utterances within the metacognition/metalinguistic category. Memory and orientation statements are defined as statements that question/ consider when events occurred or statements that attempt to reconcile what was happening within the video recording and/or when it happened. These statements fill in information for the coach or the participants to provide context regarding the video clip. They can also be self-orienting comments that add details to what is happening in the video clip, such as mentioning what day of the week the video was taken, specifying who "he or she" is referring to in the clip, or telling a short background story. These statements accounted for $64 \%$ of Joe's metacognitive statements and $59 \%$ for Sharon. Examples from sessions early and late in the intervention period are included in Figure 7.

\section{b) Understanding and managing TBI behaviors}

239 of the 539 metacognition/metalinguistic utterances (44\%) were coded as understanding and managing TBI behaviors. Statements included within this subcategory discuss impairments associated with TBI or suggest ways to manage the situation. This includes explaining the anatomical underpinnings of challenges that the participant with TBI is experiencing or providing suggestions on how to manage challenges occurring due to deficits related to the participant's TBI (e.g. writing in a planner, setting a timer, formatting questions so they are multiple choice). Twenty percent of Joe's metacognitive statements and $15 \%$ of Sharon's were within this category. Coaches devoted $91 \%$ of their metacognitive statements to this topic.

\section{c) Self-explanation}

These statements include 49 of the 539 utterances (9\%) within the discourse behaviors category. A self-explanation is defined as a statement that attempts to justify one's actions. These statements often start with, "I did that because..." This accounted for $20 \%$ of both Joe and Sharon's metacognitive statements, whereas it was $<1 \%$ for coaches $(n=1)$.

\section{d) Discussion of VSM approach}

This subcategory includes 26 of the 539 (5\%) metacognition/ metalinguistic utterances. This subcategory contains statements that talk about the purpose and steps of VSM. This includes explaining the steps as well as verifying that the purpose of watching the recorded conversations is to reflect and improve on all interactions. These statements accounted for $4-6 \%$ of metacognition statements across participants and coaches.

\section{Logistics}

Logistics is a category of utterances that includes statements that discuss challenges that occurred with the iPod or are statements that are tips on how to use the iPod. This category includes of 27 out of the 4,783 (<1\%) total utterances. Common logistics statements discuss managing the battery of the iPod or finding a good environment to record a conversation in where there is not a lot of background noise.

\section{Uncodable}

Statements that were incomplete due to unintelligibility or interruptions as well as statements that had no interpretable meaning were labeled as uncodable. This category includes 1,621 of $6,642(24 \%)$ statements.

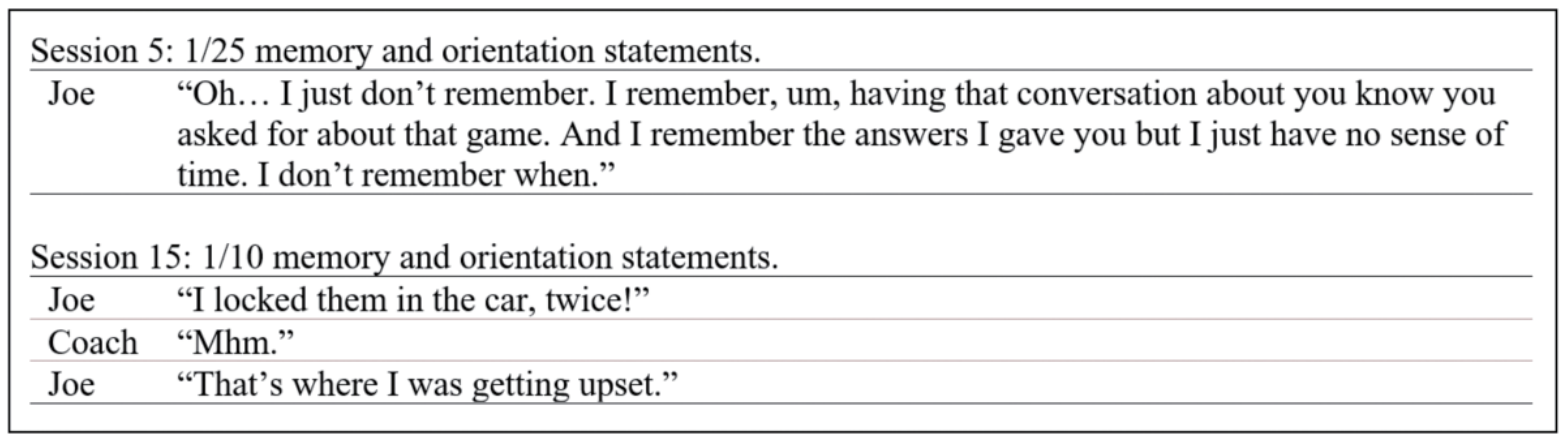

Figure 7. Memory and orientation statements. 


\section{DISCUSSION}

Joint, guided reflection on conversational behaviors through video self-modeling appears to hold promise as a means of facilitating self-assessment and altering self-regulation for individuals with TBI and their close partners. The present study establishes the feasibility of implementing such an approach with a single dyad, while highlighting elements of implementation for clinicians and researchers to focus on improving. Detailed analysis of implementation and outcomes provide a starting point for future investigations. The present investigation answers the call for feasibility studies and process-oriented research extended by Sohlberg and colleagues [68]. Adjustments that were intuitive to clinicians drove use of prompting levels. While more data is needed to determine optimal prompting sequences or levels, these preliminary results provide some indications of prompts that yield or fail to yield responses. In particular, L2 prompts appear less effective in eliciting judgments.

Discrepancy scores on the LCQ, between self and proxy ratings by individuals with TBI and close partners respectively, have been used as indicators of self-awareness and metacognition $[6,22,37]$. While the close others' ratings remained stable, ratings by the individual with TBI increased, suggesting more awareness. Further, his ratings were higher than his spouses at baseline and post-intervention intervals. Specifically, he rated himself harshly on items within the Struchen et al. [22] factors of Disinhibition/Impulsivity and Conversational Effectiveness, which relate to social communication. Individuals with TBI more frequently identify more tangible and observable factors such as Initiation/Conversation Flow behaviors $[6,21,22]$. Focusing on such behaviors (related to disinhibition/impulsivity and conversational effectiveness) through direct video review appears to have contributed to this increased sensitivity. While the increase from 84 to 101 on LCQ total scores may indicate improved self-awareness, including increased sensitivity to social communication behaviors and their effects on his communication partner, his high ratings at both increments may indicate excessively self-critical judgments. A potential consideration regarding prompting reflections on communication struggles versus only positive behaviors is the potential consequences related to self-efficacy and negative emotions. Joe's judgments became increasingly self-critical as his awareness improved. If clinicians prompt self-assessment on communication struggles, they must be prepared to address such consequences and/or make appro- priate referrals. It is also worth noting that Joe may be unique in his response to improved awareness. Sawchyn et al. [74] found that some individuals with TBI underestimate their ability, presenting as deficit-focused, and emotionally distressed. However, it is worth noting that such individuals typically have mild TBI and less substantial impairment than Joe.

Improvements were seen on the mMPC and mMSC across both individuals. Increases in MMPC interaction scores for the individual with TBI from 2.5 to 3.0/4.0 suggest improved engagement, turn taking, and pragmatic abilities. Likewise, improvements from 2.0 to $2.75 / 4.0$ on the mMPC transaction scale are indicative of improved restraint, despite continued problems with discourse cohesion organization. While mMSC acknowledging competence remained relatively stable for the partner (3.0 at baseline to 3.125 post-intervention), these scores suggest that the partner values interactions with her husband and sees him as a competent person. Improvements in mMSC revealing competence, from 2.17 to 2.88 /4.0 was evidence of increased support with topic transitions and redundancy to support comprehension. Consistent with prior research, mMPC scores increased as mMSC support scores increased [65]. Even though both participants experienced improvements, it is important to note that their behaviors during these conversations were not consistent with everyday behaviors noted within videos of conversations recorded in the home or even their interactions in most sessions. During those videos, there were frequent arguments and disagreements, the product of less restraint on the part of the individual with TBI and more sabotage on the part of the partner. Note that those videos were not transcribed and analyzed, as that was not the intent of this investigation. The effectiveness of the dyad's baseline conversation behaviors and improvements post-intervention are evidence of their ability to modify their own behaviors when the situation calls for more control. Joe even occasionally acknowledged that while he could control his outbursts during sessions, he could not sustain that restraint all the time. One potential reason for improved performance during the baseline and post-intervention conversations is that they both took place within the clinic context, rather than at home or in the community as was the case for videos reviewed in sessions. This was done to increase control for those interactions but may have inadvertently resulted in less representative interactions. That being said, the effective performance by both participants suggests that they are aware enough of their behaviors to control them, given the right context. 
The framework or protocol established for this modified VSM intervention was successful in generating opportunities for self-assessment. Because the conversations being reviewed were generated in their home and community, interactions and environments were authentic [51,54]. Participants stayed on task and for the most part were successful in reaching accurate judgments, given the support of clinicians. While the intent was to provide equal opportunities for self-reflection, the individual with TBI made more than twice the number of judgments as his close partner. In the future, more attention should be placed on fostering judgments by both individuals. At the outset, coaches set out to make as few judgments of participant behaviors as possible, however; they made nearly 100 judgments. Most of those judgments either reiterated participant judgments or came at the end of a sequence of prompts that yielded no judgment by the individual with TBI or partner. In this manner, those judgments served to close the topic and move on. Both participants reached accurate judgments when effectively supported with repeated opportunities to review video.

Prompts within the inverted hierarchy were generally effective (52-61\% accurate) in eliciting judgments, with the exception of L2 or time constrained prompts. Simply shortening the duration of the video segment for review and viewing again did not prove adequate for eliciting judgments (29\% response accuracy). As such, coaches used these prompts rarely ( $7 \%$ of instances). Treatment fidelity for the broad to direct prompting protocol was poor, at $29.27 \%$ accuracy. Following clinical decision-making principles, L2 prompts and strict adherence to the sequence from L1-L4 (broadest to most direct) do not appear to be valued. Eliminating those constraints from the protocol would be consistent with principles of implementation science identified by Sohlberg and colleagues [68]. Regardless, providing prompts with the support of video review resulted in few errors, as Joe and Sharon reached accurate judgments in $75 \%$ and $82 \%$ of opportunities respectively. This addresses Ylvisaker's [51] call for an intervention that fosters errorless self-assessment.

Although the focus of the interventions was on recognizing positive and troublesome social communication behaviors in video recorded conversations, given guided review, the intervention provided a context for conversations about conversations. While all interactants remained on task, evidence of adaptive and maladaptive discourse behaviors were both identified within the investigation. Even the partner engaged in some off-task behaviors and maladaptive discourse, con- sistent with dyad interactions examined by Body and Parker [3]. In both cases, partners used adaptive supports to redirect interactions but also reinforced maladaptive behaviors at times. As such, the intervention provided a contextualized opportunity for feedback and natural consequences of various discourse behaviors. While coaches engaged almost exclusively in adaptive discourse behaviors, both participants used adaptive and maladaptive discourse. Those maladaptive behaviors elicited a reciprocal response or consequence on the part of the partner. Training partners to resist their "righting reflex" appears to be crucial in reducing their tendency to feed into and reciprocate perseverations on interactions. The fact that the partner was able to avoid such perseverations in the post-intervention conversation suggests some awareness. Further, the partner acknowledged her tendency to set-up or sabotage interactions, in spite of her knowledge of the consequences (outbursts and blow-ups). It should be noted that when codes were tracked for frequency per session, there were no clear trends related to increases in adaptive discourse or decreases in maladaptive discourse behaviors over time. Rather, there were fluctuations indicating that frequency counts varied by the context of interactions. Because frequency counts do not necessarily directly serve as indicators of more positive or less positive behaviors, macro measures of social pragmatics and discourse (i.e., LCQ -self and -other ratings, MPC and MPC ratings) were used as outcome measures instead.

This modified VSM protocol fosters discussions of metacognition and metalinguistics as it relates to social communication exchanges. Joe identified factors that contribute to his outbursts and discussed strategies to avoid them. Likewise, Sharon admitted to using sabotage or set-ups and discussed consequences of those behaviors. Memory and orientation statements are an interesting serendipitous contribution of this investigation. Although investigators did not set out to retrain memory, both Joe and his spouse reported changes to his everyday memory functions. Joe demonstrated an improvement in immediate memory on the RBANS from pre- to post- that was sustained at a 6-month follow-up (Index score of 57 ( 0.2 percentile) at baseline, 73 (4th percentile) post-intervention, and 81 (10th percentile) at follow-up). Note that delayed memory actually declined across these intervals (index score of 75 [5th], 71 [3rd], and 64 [1st percentile] respectively). While evidence of change in this standardized measure is not convincing, orientation and memory statements were common for Joe and Sharon. Further, the specificity of 
such statements increased over time. This may be the product of reviewing videos that were recorded one to five days in advance of sessions over the course of 16 weeks. Discussions related to understanding and managing TBI behaviors were also common. In fact, these types of discussions accounted for $91 \%$ of coaches' metacognition and metalinguistic statements. The discussion-based format of the intervention provides a contextualized opportunity to carry on such discussions.

\section{CONCLUSIONS}

The modified VSM procedure described in this preliminary investigation resulted in outcomes that suggest it may be an effective tool for altering self-awareness and self-regulation within conversational interactions between persons with TBI and everyday partners. The structure of the interaction appears to keep the dyad on task, engaging in verbal reflections on their own performance. Both participants were able to make accurate judgments regarding their own interactions, given supportive cues and repeated review of video segments. Observational measures of discourse behaviors pre- and postintervention suggest that both individuals demonstrated gains in self-awareness and self-regulation within conversational discourse. While the context for the pre- and post-intervention conversations may not have been as authentic or complex, discrepancies between everyday interactions and those conversations suggest that both individuals can alter their interactions when they know they are being recorded and/or within supportive contexts. Translating that self-regulation to everyday, authentic contexts may require further interventions but even changes within those controlled contexts is promising.

\section{Limitations and future directions}

The present investigation was preliminary, limiting the ability to generalize these results to other dyads with TBI. However, heterogeneity across individuals with TBI and their partners inherently calls for individualization of interventions. The detailed qualitative data collected across sessions provides some insights into future revisions to a prompting protocol, which may serve as guidance for more controlled experimental research with larger numbers of participants. The framework for intervention provides a context to consider the relative benefits and risks of prompting self-assessments of communication struggles versus positive behaviors. If we agree that
Joe's increasingly self-critical judgments are a reflection of improved self-awareness, we must consider whether prompting reflections on communication struggles is an appropriate way to change that awareness. Ownsworth and colleagues $[49,50]$ have employed MST within functional tasks such as meal preparation and volunteer work, where the focus was on increasing awareness of errors. These interventions changed awareness of errors within task and reduced errors. However, there were no changes to global awareness. Presently, it is unclear what the consequences, along with benefits, of altering global awareness may be on psychosocial status. We may need to proceed cautiously when fostering more global awareness of self within social interaction context. Ownsworth and colleagues [75] identified several awareness typologies including good self-awareness, poor self-awareness, high defensiveness, and high symptom reporting groups. The authors stress that intervention approaches for those different types of individuals must be individualized. Joe may fall within a high symptom reporting group, of deficit-focused and emotionally distressed respondents [74,75]. Managing his development of self-awareness and regulation may require a unique, individualized set of supports that nurtures positive self-assessments.

Future research should examine these questions more systematically and with larger numbers of participants. Singlesubject methodologies hold the potential to retain some of the individualization of interventions while capitalizing on systematic elements of the intervention, so that more direct statistical comparisons can be employed. An ABC method could more systematically measure changes between baseline, intervention, and maintenance phases. A multiple baselines method could be used to assure that change was not simply a product of interacting with the coaches. Finally, goal attainment scaling could be employed to measure strength of outcomes related to participant goals.

\section{ACKNOWLEDGMENTS}

The authors would like to acknowledge the tireless work of our research transcribers, Hannah Marthaler and Kristen Eul. Further, research assistants Lian Arzbecker, Kelsey Weberg, and Jessica Ong compiled and transferred all qualitative data points to code books for tabulation. This research was supported by a summer research grant from the University of Wisconsin - Eau Claire Office of Research and Sponsored Programs, as well as a Blugold Fellowship. A grant from the Cen- 
ter for Excellence in Teaching and Learning provided iPods for this and related research. Finally, the authors wish to thank our participants for their hard work and dedication to the intervention.

\section{REFERENCES}

1. Kreuter M, Sullivan M, Dahloff AG, Siosteen A. Partner relationships, functioning, mood, and global quality of life in persons with spinal cord injury and traumatic brain injury. Spinal Cord. 1998; 36:252-261.

2. Liss M, Willer B. Traumatic brain injury and marital relationships: a literature review. International Journal of Rehabilitation Research. 1990;13:309-320.

3. Body R, Parker M. Topic repetitiveness after traumatic brain injury: an emergent, jointly managed behaviour. Clinical Linguistics \& Phonetics. 2005;19(5):379-392.

4. Coelho CA, Youse KM, Le KN. Conversational discourse in closedhead-injured and non-brain-injured adults. Aphasiology. 2002; 16(4/5/6):659-672.

5. Cools C, Manders E. Analysis of language and communication function in traumatic brain injured patients. International Journal of Rehabilitation Research. 1998;21:323-329.

6. Douglas JM. Relation of executive functioning to pragmatic outcome following severe traumatic brain injury. Journal of Speech, Language, and Hearing Research. 2010;53(2):365-382.

7. Galski T, Tompkins C, Johnston MV. Competence in discourse as a measure of social integration and quality of life in persons with traumatic brain injury. Brain Injury. 1998;12(9):769-782.

8. McDonald S, Pearce S. Requests that overcome listener reluctance: impairment associated with executive dysfunction in brain injury. Brain and Language. 1998;61(1):88-104.

9. Milton SB, Wertz RT. Management of persisting communication deficits in patients with traumatic brain injury. In B.P. Uzzell \& Y. Gross (Eds.), Clinical neuropsychology of intervention. Boston: Martinus Nijhoff Publishing. 1986.

10. Fleming J, Strong J. A longitudinal study of self-awareness: functional deficits underestimated by persons with brain injury. Occupational Therapy Journal of Research. 1999;19(1):3-17.

11. Hart T, Sherer M, Whyte J, Polansky M, Novack TA. Awareness of behavioral, cognitive, and physical deficits in acute traumatic brain injury. Archives of Physical Medicine and Rehabilitation. 2004;85(9):1450-1456.

12. Sherer M, Boake C, Levin E, Silver BV, Ringholz G, High Jr WM. Characteristics of impaired awareness after traumatic brain injury. Journal of the International Neuropsychological Society. 1998; 4(4):380-387.

13. Sherer M, Hart T, Nick TG. Measurement of impaired self-awareness after traumatic brain injury: a comparison of the patient competency rating scale and the awareness questionnaire. Brain Injury. 2003;17(1):25-37.

14. Bond F, Godfrey HPD. Conversation with traumatically brain in- jured individuals: a controlled study of behavioural changes and their impact. Brain Injury. 1997;11(5):319-330.

15. Angeleri R, Bosco FM, Zettin M, Sacco K, Colle L, Bara BG. Communicative impairment in traumatic brain injury: a complete pragmatic assessment. Brain and Language. 2008;107(3):229-245.

16. Babbage DR, Yim J, Zupan B, Neumann D, Tomita MR, Willer B. Metaanalysis of facial affect recognition difficulties after traumatic brain injury. Neuropsychology. 2011;25(3):277-285.

17. McDonald S. Impairments in social cognition following severe traumatic brain injury. Journal of the International Neuropsychological Society. 2013;19(03):231-246.

18. Watts AJ, Douglas JM. Interpreting facial expression and communication competence following severe traumatic brain injury. Aphasiology. 2006;20(8):707-722.

19. Zupan B, Babbage D, Neumann D, Willer B. Recognition of facial and vocal affect following traumatic brain injury. Brain Injury. 2014;28(8):1087-1095.

20. Ylvisaker M, Szekeres SF, Henry K, Sullivan DM, Wheeler P. Topics in cognitive rehabilitation therapy; In M. Ylvisaker, EMR Gobble (Eds.), Community re-entry for head injured adults (p. 137-220). Boston: College-Hill Press/Little Brown \& Co. 1987.

21. Bracy CA, Douglas JM. Marital dyad perceptions of injured partners' communication following severe traumatic brain injury. Brain Impairment. 2005;6(1):1-12.

22. Struchen MA, Pappadis MR, Mazzei DK, Clark AN, Davis LC, Sander AM. Perceptions of communication abilities for persons with traumatic brain injury: Validity of the La Trobe Communication Questionnaire. Brain Injury. 2008;22(12):940-951.

23. Grice HP. Logic and conversation. In P. Cole and J.L. Morgan (Eds.), Syntax and semantics: Vol. 3: Speech Acts (p. 41-58). Academic Press: New York. 1975.

24. Le K, Mozeiko J, Coelho C. Discourse analyses: characterizing cognitive communication disorders following TBI. The ASHA Leader. 2011;16(2):18-21.

25. Martin I, McDonald S. Weak coherence, no theory of mind, or executive dysfunction? Solving the puzzle of pragmatic language disorders. Brain and Language. 2003;85(3):451-466.

26. Togher L, McDonald S, Coelho CA, Byom L. Cognitive communication disability following TBI. In S. McDonald, L. Togher, C. Code (Eds.), Social and communication disorders following traumatic brain injury (2nd ed., p. 89-118). East Sussex: Psychology Press. 2014.

27. Godfrey HPD, Shum D. Executive functioning and the application of social skills following traumatic brain injury. Aphasiology. 2000; 14(4):433-444.

28. Godfrey HPD, Knight RG, Bishara SN. The relationship between level of social skill and family problem-solving interaction following very severe closed head injury. Brain Injury. 1991;5:207-211.

29. Hoepner JK. Characteristics of effective communication partners in supporting persons with traumatic brain injury. University of Wisconsin - Madison; Madison, WI: Dissertation Abstracts International. 2010.

30. Paterson J, Stewart J. Adults with acquired brain injury: percep- 
tions of their social world. Rehabilitation Nursing. 2002;27(1):1338.

31. Douglas J, Bracy C, Snow P. Measuring perception of communicative ability: Reliability and Validity of the La Trobe Communication Questionnaire. Journal of Head Trauma Rehabilitation. 2007; 22(1):31-38.

32. Douglas JM, Bracy CA, Snow PC. Exploring the factor structure of the La Trobe Communication Questionnaire: insights into the nature of communication deficits following traumatic brain injury. Aphasiology. 2007; 21(12):1181-1194.

33. Dahlberg C, Hawley L, Morey C, Newman J, Cusick CP, HarrisonFelix C. Social communication skills in persons with post-acute traumatic brain injury: three perspectives. Brain Injury. 2006; 20(4):425-435.

34. Fiske ST. Attention and weight in person perception: the impact of negative and extreme information. Journal of Personality and School Psychology. 1980;39:889-906.

35. Halford WK, Keefer E, Osgarby SM. "How has the week been for you two?" Relationship satisfaction and hindsight memory biases in couples' reports of relationship events. Cognitive Therapy and Research. 2002;26(6):759-773.

36. Matt GE, Vázquez C, Campbell WK. Mood-congruent recall of affectively toned stimuli: a meta-analytic review. Clinical Psychology Review. 1992;12(2):227-255.

37. Hoepner JK, Turkstra LS. Video-based administration of the la trobe communication questionnaire for adults with traumatic brain injury and their communication partners. Brain Injury. 2013;27(4):464-472.

38. Fleming JM, Strong J, Ashton R. Cluster analysis of self-awareness levels in adults with traumatic brain injury and relationship to outcome. Journal of Head Trauma Rehabilitation. 1998;13(5):3951.

39. Toglia JP. The dynamic interactional model of cognition in cognitive rehabilitation. In N. Katz (Ed.), Cognition, occupation, and participation across the lifespan: Neuroscience, neurorehabilitation and models of intervention in occupational therapy (Vol. 3, p. 161-203). Bethedsa: MD: The American Occupational Therapy Association. 2011.

40. Toglia J, Kirk U. Understanding awareness deficits following brain injury. NeuroRehabilitation. 2000;15(1):57-70.

41. Ownsworth T, Desbois J, Grant E, Fleming J, Strong J. The associations among self-awareness, emotional well-being, and employment outcome following acquired brain injury: a 12-month longitudinal study. Rehabilitation Psychology. 2006;51(1):50-59.

42. Burke WH, Zencius AH, Wesolowski MD, Doubleday F. Improving executive function disorders in brain-injured clients. Brain Injury. 1991;5:241-252.

43. Cicerone KD, Giacino JT. Remediation of executive function deficits after traumatic brain injury. NeuroRehabilitation. 1992;2:1222.

44. Cicerone KD, Wood JC. Planning disorder after closed head injury: a case study. Archives of Physical Medicine and Rehabilitation. 1987;68(2):111-115.
45. Levine B, Robertson IH, Clare L, Carter G, Hong J, Wilson BA, et al. Rehabilitation of executive functioning: an experimental-clinical validation of goal management training. Journal of International Neuropsychological Society. 2000;6:299-312.

46. Turkstra LS, Flora TL. Compensating for executive function impairments after TBI: a single case study of functional intervention. Journal of Communication Disorders. 2002;35:467-482.

47. Sohlberg MM, Ehlhardt L, Kennedy M. Instructional techniques in cognitive rehabilitation: a preliminary report. Seminars in Speech and Language. 2005;26:268-279.

48. von Cramon DY, Matthes-von Cramon G, Mai N. Problem solving deficits in brain injured patients. A therapeutic approach. Neuropsychological Rehabilitation. 1991;1:45-64.

49. Ownsworth T, Quinn H, Fleming J, Kendall M, Shum D. Error selfregulation following traumatic brain injury: a single case study evaluation of metacognitive skills training and behavioural practice interventions. Neuropsychological Rehabilitation. 2010;20(1): 59-80.

50. Ownsworth T, Fleming J, Desbois J, Strong J. Kuipers P. A metacognitive contextual intervention to enhance error awareness and functional outcome following traumatic brain injury: a single case experimental design. Journal of the International Neuropsychological Society. 2006;12:54-63.

51. Ylvisaker M. Self-coaching: a context-sensitive, person-centered approach to social communication after traumatic brain injury. Brain Impairment. 2006;7(3):246-258.

52. Fasotti L, Kovacs F, Eling PATM, Brouwer WH. Time pressure management as a compensatory strategy training after closed head injury. Neuropsychological Rehabilitation. 2000;10:47-65.

53. Togher L, McDonald S, Tate R, Power M, Rietdijk R. Training communication partners of people with severe traumatic brain injury improves everyday conversations: A multicenter single blind clinical trial. Journal of Rehabilitation Medicine. 2013;45:637-645.

54. Youse KM, Coelho CA. Treating underlying attention deficits as a means for improving conversational discourse in individuals with closed head injury: a preliminary study. NeuroRehabilitation. 2009;24(4):355-364.

55. Baker SD, Lang R, O'Reilly M. Review of video modeling with students with emotional and behavioral disorders. Education and Treatment of Children. 2009;32(3):403-420.

56. Buggey T, Ogle L. Video self-modeling. Psychology in the Schools. 2012;49(1):52-70.

57. Buggey T. A picture is worth video self-modeling applications at school and home. Journal of Positive Behavior Interventions. 2007;9(3):151-158.

58. Cream A, O'Brian S, Jones M, Block S, Harrison E, Lincoln M, et al. Randomized controlled trial of video self-modeling following speech restructuring treatment for stuttering. Journal of Speech, Language, and Hearing Research. 2010;53(4):887-897.

59. Lang R, Shogren KA, Machalicek W, Rispoli M, O’Reilly M, Baker S, et al. Video self-modeling to teach classroom rules to two students with Asperger's. Research in Autism Spectrum Disorder. 2009;3(2): 483-488. 
60. Ortiz J, Burlingame C, Onuegbulem C, Yoshikawa K, Rojas ED. The use of video self- modeling with English language learners: implications for success. Psychology in the Schools. 2012;49(1): 23-29.

61. Prater MA, Carter N, Hitchcock C, Dowrick P. Video self-modeling to improve academic performance: a literature review. Psychology in the Schools. 2012;49(1):71-81.

62. Magill-Evans J, Harrison MJ, Benzies K, Gierl M, Kimak C. Effects of parenting education on first-time fathers' skills in interactions with their infants. Fathering. 2007;5(1):42.

63. Meharg SS, Lipsker LE. Parent training using videotape self-modeling. Child \& Family Behavior Therapy. 1992;13(4):1-27.

64. Creer TL, Miklich DR. The application of a self-modeling procedure to modify inappropriate behavior: a preliminary report. Behaviour Research and Therapy. 1970;8(1):91-92.

65. Hoepner JK, Sell L, Kooiman H. Case study of partner training in corticobasal degeneration. Journal of Interactional Research in Communication Disorders. 2015;6(2):157-186.

66. Miller WR, Rolnick S. Motivational interviewing: helping people change. New York. NY: Guilford Press. 2012.

67. Greene JC, Caracelli VJ, Graham WF. Toward a conceptual framework for mixed-method evaluation designs. Educational Evaluation and Policy Analysis. 1989;11(3):255-274.

68. Sohlberg MM, Kucheria P, Fickas S, Wade SL. Developing brain injury interventions on both ends of the treatment continuum depends upon early research partnerships and feasibility studies.
Journal of Speech, Language, and Hearing Research. 2015;58(6): S1864-S1870.

69. Randolph C. RBANS Manual-Repeatable Battery for the Assessment of Neuropsychological Status. San Antonio, TX, Psychological Corp (Harcourt). 1998.

70. McKay C, Casey JE, Wertheimer J, Fichtenberg NL. Reliability and validity of the RBANS in a traumatic brain injured sample. Archives of Clinical Neuropsychology. 2007;22(1):91-98.

71. Douglas J, O'Flaherty C, Snow P. Measuring perception of communicative ability: the development and evaluation of the La Trobe communication questionnaire. Aphasiology. 2000;14(3): 251-268.

72. Kagan A, Winckel J, Black SE, Duchan JF, Simmons-Mackie N, Square P. A set of observational measures for rating support and participation in conversation between adults and their conversation partners. Topics in Stroke Rehabilitation. 2004;11(1):67-83.

73. Strauss A, Corbin J. Basics of qualitative research: grounded theory procedures and techniques. Newbury Park. CA: Sage. 1990.

74. Sawchyn JM, Mateer CA, Suffield JB. Awareness, emotional adjustment, and injury severity in postacute brain injury. Journal of Head Trauma Rehabilitation. 2005;20:301-314.

75. Ownsworth T, Fleming J, Strong J, Radel M, Chan W, Clare L. Awareness typologies, long-term emotional adjustment and psychosocial outcomes following acquired brain injury. Neuropsychological Rehabilitation. 2007;17(2):129-150. 